\section{$\underset{\substack{\text { hommes } \\ \text { \& migrations }}}{ }$}

\section{Hommes \& migrations}

Revue française de référence sur les dynamiques

migratoires

\section{2 | 2018}

Exposer les migrations

\title{
Saint-Étienne Cosmopolitaine. Retour sur un projet de territoire (2014-2016)
}

\section{Cyril Longin}

\section{OpenEdition \\ Journals}

Édition électronique

URL : https://journals.openedition.org/hommesmigrations/6629

DOI : 10.4000/hommesmigrations.6629

ISSN : 2262-3353

\section{Éditeur}

Musée national de l'histoire de l'immigration

\section{Édition imprimée}

Date de publication : 1 juillet 2018

Pagination : 55-62

ISBN : 978-2-919040-42-1

ISSN : $1142-852 X$

Référence électronique

Cyril Longin, « Saint-Étienne Cosmopolitaine. Retour sur un projet de territoire (2014-2016) », Hommes \& migrations [En ligne], 1322 | 2018, mis en ligne le 01 juillet 2020, consulté le 21 janvier 2022. URL

http://journals.openedition.org/hommesmigrations/6629; DOI : https://doi.org/10.4000/

hommesmigrations.6629 


\title{
SAINT-ÉTIENNE \\ COSMOPOLITAINE. \\ RETOUR SUR UN PROJET \\ DE TERRITOIRE (2014-2016)
}

Par CYRIL LONGIN, directeur des Archives municipales de Saint-Étienne.

\author{
Le bassin industriel de Saint-Étienne s'est construit grâce à \\ un apport de main-d'œuvre venue d'ailleurs dont les archives \\ de la ville ont conservé les traces. Pour valoriser ce fonds, \\ les Archives municipales ont accueilli entre septembre 2015 \\ et septembre 2016 l'exposition Saint-Étienne cosmopolitaine, \\ des migrations dans la ville. Conçue en partenariat avec \\ des laboratoires de recherche et des associations locales, \\ cette exposition visait à connecter les trajectoires migratoires \\ passées à la diversité des cultures qui composent \\ la population stéphanoise. Cette exposition a donné lieu \\ à une véritable saison culturelle dans la ville et inspiré \\ d'autres municipalités.
}

À l'image d'autres bassins industriels, Saint-Étienne a vu son territoire se peupler rapidement, au fil des $\mathrm{XIX}^{\mathrm{e}}$ et XX $\mathrm{XX}^{\mathrm{e}}$ siècles, avec l'arrivée de main-d'œuvre venue grossir les rangs de ses entreprises. En provenance d'Ardèche, de Haute-Loire, d'Italie, d'Espagne, de Pologne, de Grèce, d'Arménie, d'Algérie, du Maroc, de Turquie, de pays d'Afrique subsaharienne ou d'Asie..., quittant un ailleurs proche ou lointain, à différentes époques, pour différentes raisons, ces populations se sont progressivement enracinées dans un paysage en mutation.
Ces origines plurielles participent aujourd'hui de l'identité de la ville, en grande partie héritée de cette aventure industrielle et ouvrière. Les formes et les lieux d'expression de cette diversité sont aujourd'hui perceptibles dans l'espace urbain, sans que les Stéphanois aient toujours conscience de cette profondeur historique. C'est pourquoi les Archives municipales de Saint-Étienne, avec le concours du service Saint-Étienne Ville d'art et d'histoire ${ }^{1}$, ont souhaité mettre en lumière l'histoire du peuplement de Saint-Étienne. Ces deux 
structures ont imaginé Saint-Étienne cosmopolitaine, des migrations dans la ville, un projet culturel qui dresse le portrait de la ville d'aujourd'hui, telle qu'elle a été façonnée par la diversité des habitants qui la composent.

\section{La naissance d'un projet territorial}

Si la programmation des établissements culturels répond généralement à des projets construits, traduisant un projet scientifique et culturel, des projets s'imposent parfois d'eux-mêmes. C'est le cas de Saint-Étienne cosmopolitaine, né de rencontres successives dans un temps court, au début de l'année 2014. Entre janvier et mars 2014, les Archives municipales sont amenées à rencontrer divers acteurs menant des projets distincts, mais ayant un dénominateur commun : l'histoire du peuplement de la ville. Ces initiatives concomitantes étaient-elles le fruit du hasard ou le résultat du processus de patrimonialisation des migrations ? Il est

La mémoire des migrations

n’avait jamais été réellement

abordée par les établissements

culturels de la ville. Seule

une précédente exposition

au Musée de la mine,

D'ici et là-bas, consacrée aux mineurs algériens, avait été présentée au public en 2012. difficile de répondre à cette question. Mais sans doute était-il venu, à Saint-Étienne, le temps de traiter - enfin - cette question souvent jugée politiquement épineuse. Les partenaires engagés dans cette aventure ont été, au fur et à mesure de la réflexion, invités à rejoindre la dynamique. Ils sont issus de domaines et de cultures professionnelles différentes, venant aussi bien de l'université que du monde associatif. Le laboratoire Celec (Centre d'études sur les littératures étrangères et comparées, Université Jean Monnet) portait le projet SaintÉtienne plurilingue dédié à la valorisation, à travers des collectes de témoignages, de la richesse de la situation linguistique locale. Lassociation Carton plein menait le projet Sainté itinéraires croisés, destiné à faire émerger l'identité des commerces marquée par ses boutiques. Le laboratoire Cierec (Centre interdisciplinaire d'études et de recherches sur l'expression contemporaine, Université
Jean Monnet) et le CMTRA (Centre des musiques traditionnelles Rhône-Alpes) étaient engagés dans le projet Comment sonne la ville? Les musiques migrantes, visant à montrer l'importance des musiques migrantes dans les mémoires urbaines. Le Centre Max Weber (Université Jean Monnet) et l'association MIM (Migrations et images mémorielles) menaient un travail consacré aux lieux de sociabilité et aux parcours migratoires : Rendez-vous au café. C'est ainsi qu'est née Saint-Étienne cosmopolitaine, des migrations dans la ville, mêlant archives, histoire, socio-anthropologie, ethnomusicologie et sociolinguistique.

La mémoire des migrations n'avait jamais été réellement abordée par les établissements culturels de la ville. Seule une précédente exposition au Musée de la mine, D'ici et là-bas, consacrée aux mineurs algériens, avait été présentée au public en 2012. Et encore, ce travail étant le fruit d'une enquête sociologique menée par Catherine Gauthier, qui devint d'ailleurs un des acteurs de Saint-Étienne cosmopolitaine. La construction du projet s'est déroulée dans un contexte local particulier, à savoir quelques mois avant les élections municipales de 2014 ayant conduit à un changement de majorité et de bord politique. La dynamique aurait pu être remise en cause, compte tenu du caractère sensible et politique du sujet de l'immigration. Il n'en fut rien avec, au contraire, un réel encouragement, notamment de la part de l'élu en charge des affaires culturelles. Par ailleurs, l'année 2015 a été marquée par de fortes tensions nationales et internationales, qui auraient pu avoir des conséquences fâcheuses. En premier lieu, les attentats de janvier 2015 ont fait resurgir des questions liées à l'immigration, à l'intégration et aux religions devenues très présentes dans l'espace public et médiatique. Mais, surtout, la « crise des migrants » de l'été 2015, quelques semaines avant l'ouverture de l'exposition aux Archives municipales, a engendré de vives réactions dans la sphère politique nationale et locale, mettant le sujet au cœur du débat public. Gaël Perdriau, maire de Saint-Étienne, fera d'ailleurs l'objet d'importantes attaques lorsque, quelques jours avant le vernissage, il publie une tribune dans Le Figaro se déclarant favorable à l'accueil de migrants. Les 


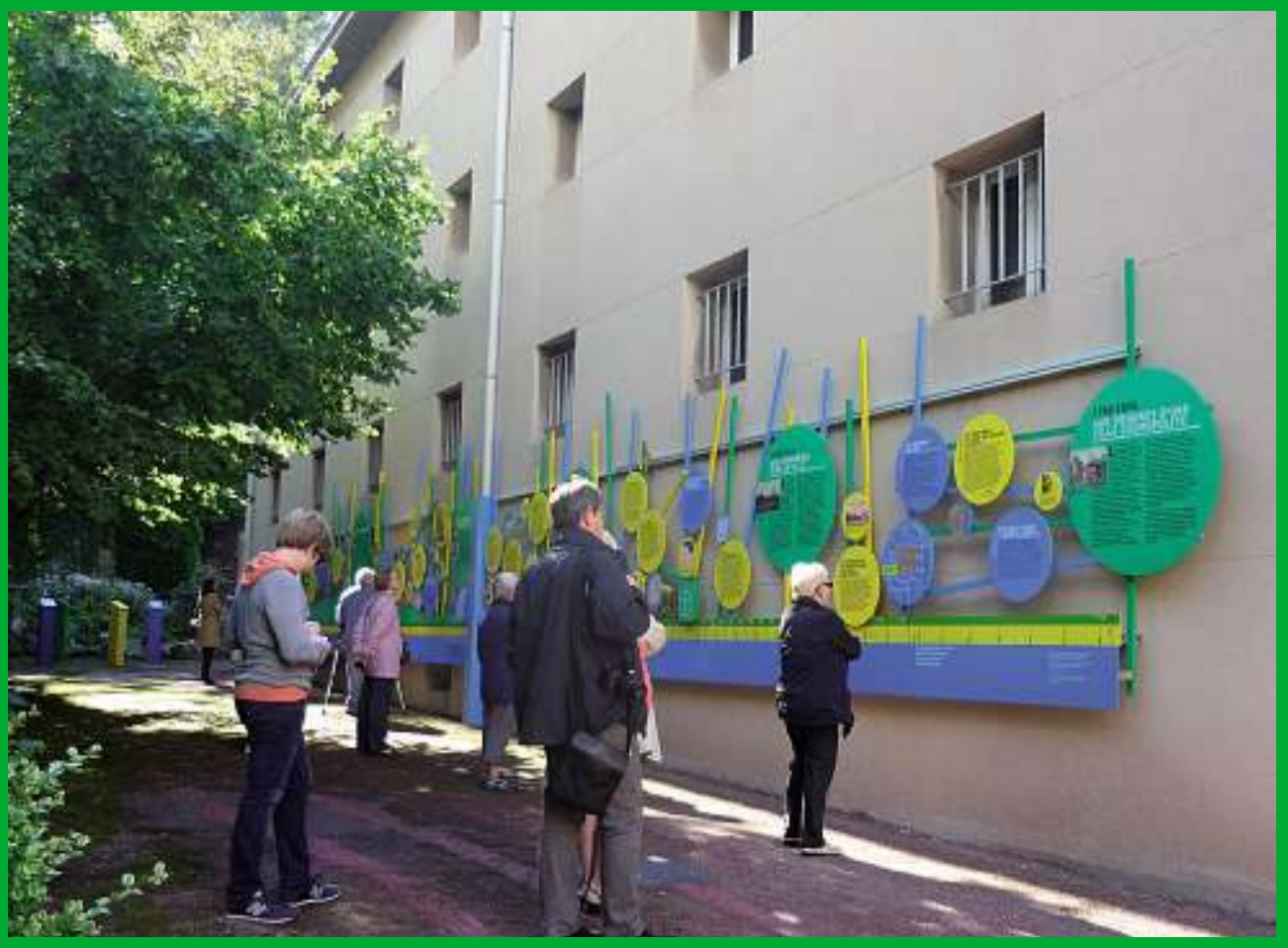

Vue de la frise chronologique des vagues de migration dans la première partie de l'exposition Saint-Étienne cosmopolitaine, des migrations dans la ville aux Archives municipales (2015-2016). Extérieur du bâtiment. @ ARCHIVES MUNICIPALES DE SAINT-ÉTIENNE.

attaques émanèrent notamment de sa propre famille politique (Les Républicains), mais également et surtout des réseaux sociaux. Son compte Facebook fut, par exemple, assailli de messages d'insultes, entraînant sa fermeture momentanée. C'est dans ce contexte de tensions, d'attaques et de couverture médiatique que s'ouvre l'exposition.

\section{Imaginer un récit à plusieurs voix}

Présentée aux Archives municipales de septembre 2015 à septembre 2016, l'exposition a constitué le point d'appui - et de ralliement - de l'ensemble des acteurs du projet interdisciplinaire. Le scénario de l'exposition, conçu par les Archives municipales, a été agrémenté des contributions des partenaires scientifiques sous une forme validée par l'ensemble des acteurs.

L'exposition s'inscrit comme un espace et un temps de découverte, de réflexion, d'échange, de transmission où se croisent des approches et des sources complémentaires. Tout d'abord, la voix des archives publiques, sources matérielles de la connaissance historique. Cependant, ces documents, produits essentiellement dans le cadre d'une procédure administrative - et souvent de surveillance -, sont forcément partiels et partiaux. Si éclairantes qu'elles soient, les archives se doivent d'être complétées et enrichies par des sources annexes. Le récit créé à partir des sources archivistiques mobilisées se traduit dans l'exposition par une frise 
chronologique, illustrant les flux migratoires à l'aune du développement industriel de la ville. Il amène également le visiteur à interroger les sources mobilisées, mais aussi leurs limites.

Ensuite, la voix des chercheurs qui travaillent sur le territoire. Elle s'appuie sur le recueil de parcours de vies d'habitants réalisé par des étudiants et des chercheurs. Les laboratoires questionnent ainsi les formes d'expression de la diversité culturelle dans l'espace urbain. Cette deuxième séquence du parcours d'exposition se traduit par la présentation des travaux en cours, notamment l'écoute d'un échantillon des collectes orales, mais aussi par le souhait d'expliciter la démarche scientifique de chaque partenaire. Il s'agit donc d'une forme de vulgarisation de la recherche scientifique, mais surtout de remettre le travail des chercheurs au cœur de la vie des citoyens.

La voix des habitants constitue enfin la dernière

Pensée dans sa globalité, Saint-Étienne cosmopolitaine avait vocation à s'adresser à un public large et divers. L'offre se devait donc d'être au plus près des attentes du public, individuel, en groupe, scolaire ou maîtrisant peu la langue française. composante de ce récit. Elle comble les lacunes des archives ou des collectes et apporte une dimension citoyenne au projet. L'expérience des habitants enrichit donc la démarche, initiée en amont de l'exposition à travers l'occupation d'une boutique éphémère dans le quartier de Jacquard. Pendant la Biennale du design 2015, les habitants ont été invités à participer au Cosmotrombinoscope ou à témoigner de leur parcours ou de celui de leur famille. Cette préfiguration du projet a permis de sensibiliser les habitants à cet aspect de leur mémoire collective et individuelle, mais également aux archives et à la nécessité d'être acteurs de leur patrimoine. Si l'opération a été positive, avec des approches bienveillantes de la part des habitants, la démarche a pu surprendre et n'a concerné que quelques dizaines de personnes. Cette participation des habitants est présente dans l'exposition grâce à la bibliothèque des projets. Cette dernière présente les projets éducatifs ou associatifs qui traitent des mémoires des migrations et pour lesquels il existe des traces dans les archives. Cette bibliothèque, se voulant participative et évolutive, a été enrichie par les projets ayant fait l'objet de dons d'archives mais également conduits dans le cadre de l'exposition.

\section{Une offre diversifiée et partenariale}

Pensée dans sa globalité, Saint-Étienne cosmopolitaine avait vocation à s'adresser à un public large et divers. L'offre se devait donc d'être au plus près des attentes du public, individuel, en groupe, scolaire ou maîtrisant peu la langue française.

La sensibilisation et la participation du public scolaire ont constitué une composante essentielle du projet. Il s'agissait aussi bien de proposer de simples visites guidées que de permettre aux élèves de tous niveaux de devenir acteurs de ce récit. Les Archives municipales ont ainsi lancé un appel à projets auprès des enseignants, notamment du primaire, visant à faire travailler les élèves sur l'héritage des migrations. Cinq classes issues de cinq écoles ont répondu à l'appel, avec des méthodologies, des approches et des restitutions propres à chacune : webradio, chansons, spectacles, exposition, etc. Des classes de lycées ont également souhaité s'inscrire dans la dynamique, notamment dans le cadre des projets Eureka portés par le Conseil régional ${ }^{2}$. La réalisation la plus emblématique est certainement la production d'un documentaire par les élèves du lycée technique Jacob Holtzer de Firminy. Ce film, Tous les chemins mènent en Ondaine, retrace les parcours de nombreux habitants enregistrés par les élèves.

Souhaitant jouer un rôle fédérateur, les Archives municipales ont proposé, pour la première fois, une programmation trimestrielle autour de l'exposition. Cette offre se devait de refléter le caractère collectif du projet en offrant la possibilité à l'ensemble des partenaires et des acteurs (institutionnels, associatifs, artistiques, etc.) de faire connaître leurs 
actions. À titre d'exemples, une pièce de théâtre, Ces inconnus chez nous, a été présentée au Musée d'art et d'industrie et un spectacle, Au Claire des rimes, a été proposé dans les médiathèques. Pendant un an, ce sont près de soixante-dix événements qui ont été proposés, dont - seulement - douze organisés par les Archives municipales, traduisant la dynamique ainsi créée. Les propositions étaient d'une grande diversité de forme et d'objet : workshop, spectacles, concerts, colloques, expositions, marches urbaines, projections, ateliers, lectures, émissions de radio, etc. Une partie de ces événements a pu prendre place dans la programmation de la Biennale Traces en Rhône-Alpes en 2015. Saint-Étienne a d'ailleurs accueilli la journée d'études de Traces en octobre 2015 consacrée à 10 ans de recherches sur les mémoires d'immigration.

Conscient de l'ampleur et l'importance de la dynamique du projet, le groupe de pilotage créé a souhaité laisser une trace de Cosmopolitaine. Il s'agissait de conserver aussi bien le fruit de la recherche historique - nous partions d'une page blanche - que les traces de la mobilisation des chercheurs et des habitants. Le service Ville d'art et d'histoire, disposant d'une ligne éditoriale, a ainsi publié une brochure Laissez-vous conter Saint-Étienne cosmopolitaine reprenant une bonne part des textes de l'exposition. Édité à 5000 exemplaires, le livret a été diffusé dans l'ensemble des établissements culturels de la ville et distribué aux visiteurs de l'exposition. D'un abord simple, sans approche universitaire, la brochure a permis aux visiteurs de partir de l'exposition avec une trace. Elle fait encore figure de référence sur le sujet et elle nous est demandée régulièrement par des habitants ou des chercheurs extérieurs.

Par ailleurs, les Archives municipales ont mis à disposition leur site Internet afin de valoriser et de rendre pérennes les initiatives citoyennes présentées dans la bibliothèque des projets. Les projets présentés ont donc pris place dans la rubrique "Tranches d'histoire", composé d'articles ayant vocation à raconter la ville. Cette initiative n'est pas nouvelle pour le service: le site Internet, revu en 2014, se veut participatif et ouvert aux contributions des Stéphanois. Les nouveaux articles ont donc enrichi une démarche déjà engagée.

\section{Liste des partenaires du projet}

Institutions municipales et muséales: Médiathèques de Saint-Étienne, Cinémathèque de SaintÉtienne, Fête du livre de Saint-Étienne, Musée de la mine, Musée d'art et d'industrie, Saint-Étienne Ville d'art et d'histoire, Musée des Verts, Mémorial de la résistance et de la déportation de la Loire.

Université et recherche: Gremmos (Groupe de recherches et d'études sur les mémoires du monde ouvrier stéphanois), Institut du travail (Université Jean Monnet), Cierec (Centre interdisciplinaire d'études et de recherches sur l'expression contemporaine, Université Jean Monnet), CMTRA (Centre des musiques traditionnelles de Rhône-Alpes), Celec (Centre d'études sur les littératures étrangères et comparées, Université Jean Monnet), Centre Max Weber (Université Jean Monnet), Association MIM (Migrations et images mémorielles), Forum Traces en Rhône-Alpes.

Associations : Carton plein, Centre social du Babet, École de l'oralité, Association Vivre à Beaubrun Tarentaize, Radio Dio.

Compagnies artistiques: Compagnie Cour d'art \& co, Compagnie Zangmo, Compagnie Dire d'étoile, Compagnie Collectif X.

Éducation nationale: lycée Jacob Holtzer de Firminy, lycée Étienne Mimard de Saint-Étienne, lycée Claude Fauriel de Saint-Étienne, école primaire du Soleil, école primaire Soleysel, école privée Saint-Ennemond.

\section{Une reconnaissance du public et... publique}

La perception du public constituait une grande interrogation vu le contexte. Nous pouvions même craindre un temps des réactions hostiles, voire même des dégradations tant les tensions étaient fortes ${ }^{3}$. Mais il n'en fut rien, et l'accueil du public fut à la hauteur de l'investissement. L'exposition a reçu près de 3500 visiteurs, chiffre plus qu'honorable pour les Archives municipales si on tient compte des 
spécificités de cet établissement: fermeture les week-ends, éloignement du centre-ville, déficit de popularité, par rapport aux musées notamment, etc. Mais un élément a retenu notre attention : l'exceptionnelle importance du nombre de demande de visites de groupes, de toutes provenances. Plusieurs dizaines de visites guidées ont été assurées, du centre d'alphabétisation au Lions club, témoignant de l'universalité du sujet. La scénogra-

L'exposition a reçu près de 3500 visiteurs, chiffre plus

qu'honorable pour les

Archives municipales si on tient compte des spécificités de cet établissement :

fermeture les week-ends, éloignement du centre-ville, déficit de popularité, par rapport aux musées notamment, etc. phie, particulièrement dynamique et attractive, a sans doute joué un rôle important dans ce succès. En revanche, il est impossible de donner une estimation de la fréquentation des nombreux événements proposés pendant la saison Saint-Étienne cosmopolitaine, mais elle a certainement doublé celle de l'exposition.

Le vernissage de l'exposition en septembre 2015 coïncidait avec un afflux exceptionnel de migrants. Les médias ne purent alors que s'y intéresser. Pour autant, et afin d'éviter toute suspicion d'une opération d'opportunisme politique, il a fallu rappeler à de nombreuses reprises que le service travaillait depuis 18 mois sur ce projet. Avec une certaine amertume $e^{4}$, nous avons constaté un intérêt nouveau des médias pour les archives, avec un nombre de sollicitations bien supérieur à la normale. Le retour de la critique fut plus que favorable, mettant en avant le dynamisme de la scénographie et la volonté de relater des faits, sans jugement ni parti pris. De fait, l'actualité a fortement influencé le regard des médias, mais également celui des élus. En effet, la sensibilité du sujet et le contexte ont engendré de la part du cabinet du maire un suivi particulier et une présence systématique de l'élu à la culture lors des déplacements d'un média. À dessein : pour distinguer les discours politique et scientifique. D'une certaine manière, il s'agissait de mettre en avant la dimension scientifique du projet et se garder de toute accusation partisane. Pour autant, la dimension, et pourrait-on dire l'usage, politique de l'exposition n'est pas à négliger. Le portage du projet par les Archives municipales, équipement à la notoriété limitée mais dont la démarche scientifique ne peut être remise en cause, a sans doute facilité la communication politique. Un portage par un des deux musées, avec une bien plus grande visibilité, aurait sans doute suscité beaucoup plus de débats.

La reconnaissance publique s'est également matérialisée par un soutien inédit de la région RhôneAlpes et de la Direction régionale des affaires culturelles (DRAC) dans le cadre de l'appel à projet "Mémoires du XXe siècle». La subvention de $20000 €$, bien supérieure que pour les projets précédents, témoigne de la solidité de la démarche. Les financeurs ont notamment souligné le rôle fédérateur des archives, moteur d'une réelle dynamique. Enfin, une délégation de la ville de ClermontFerrand composée d'élus et de responsables de la direction des affaires culturelles s'est rendue pendant une journée à Saint-Étienne afin d'échanger sur le projet. Souhaitant s'engager dans une démarche proche, nos interlocuteurs envisageaient de s'inspirer de Saint-Étienne cosmopolitaine, notamment dans sa capacité à mobiliser un large pan des acteurs de la ville.

\section{Une préfiguration des archives de demain?}

En novembre 2016, une journée de bilan du projet s'est tenue à la cinémathèque de Saint-Étienne, en présence des partenaires et du public qui nous ont suivis. Cet événement avait une double vocation : réunir les acteurs d'un projet collectif, qui se connaissaient pour certains, mais aussi et surtout faire un bilan et envisager les perspectives. Car, audelà des résultats, la démarche avait suscité des attentes de la part des structures associées. Il 
s'agissait de concevoir les suites à donner à la dynamique enclenchée.

Les Archives municipales ont joué un rôle central dans la bonne marche du projet: ce lieu de ressources archivistiques a accueilli une exposition à la fois vitrine et point d'appui scientifique et lien entre de nombreux acteurs. Les archives ont pu assumer ce rôle qui a probablement permis la réussite de Saint-Étienne cosmopolitaine. Au-delà de la mise en cohérence du récit et des actions, le service a su mettre en relation des partenaires qui ne se connaissaient pas et ainsi faciliter des coopérations inédites.

Mais, du point de vue de la temporalité, sont apparues les limites d'un projet collectif consacré à un sujet à forte charge émotionnelle. Le temps des structures associatives et militantes n'est pas celui des établissements culturels. Quand ces derniers terminent un projet et passent à un autre, les associations, elles, poursuivent leurs actions et leur engagement. Les approches et les attentes sont également différentes. Il peut ainsi y avoir un important décalage entre les souhaits des différents acteurs. Tout d'abord entre les "patrimoniaux » et les universitaires. La collaboration entre les deux institutions fut particulièrement riche mais également semée d'embûches : la nécessité de vulgariser le travail universitaire (textes courts, sans jargon, etc.) ou le respect des délais et des cadres de l'exposition. Le fossé existe aussi, et sans doute de manière plus aiguë, entre les institutions publiques et les associations militantes. La construction de la programmation a révélé des divergences quant aux objectifs mêmes du projet. La démarche de 
l'institution n'est pas militante mais scientifique et patrimoniale. À ce titre, toutes les actions des acteurs n'avaient pas vocation à intégrer la programmation de Saint-Étienne cosmopolitaine. Par exemple, les manifestations de soutien aux Roms ou aux réfugiés ont suscité le débat, l'équipe des archives pensant qu'elles n'y avaient pas leur place, ne se rapportant pas directement à la question des mémoires des migrations. Les archives ont dû finalement arbitrer, revendiquant une posture institutionnelle se voulant garante de l'objectivité scientifique. Ce point avait été
Ce projet fut précurseur sur de nombreux points :

le travail sur l'héritage des migrations, la réinterrogation et la collecte de sources,

la fédération de nombreux acteurs et une programmation inédite. discuté et validé avec les partenaires scientifiques et fut finalement accepté par les associations militantes qui ont compris que ce positionnement traduisait avant tout le statut municipal des archives, soumis à une tutelle politique.

Enfin, les Archives municipales constituent un équipement culturel à vocation patrimoniale certes, mais dont l'objet reste avant tout administratif. Ses capacités humaines et financières ne sont pas adaptées pour multiplier des projets de cette ampleur. La coordination générale, aussi bien de l'exposition que de la programmation, a mobilisé, au-delà de ses capacités, une équipe réduite à deux postes à temps plein. Peu relayées par les autres institutions culturelles de la ville, les archives disposaient de faibles ressources au regard des fortes attentes extérieures.

\section{Conclusion}

Sur un thème d'actualité, interrogeant l'histoire et la mémoire, l'exposition Saint-Étienne cosmopolitaine a su mobiliser un réseau exceptionnel de partenaires pour produire une programmation riche et variée. Elle a obtenu une reconnaissance des pouvoirs publics et a suscité une forte adhésion d'un public large. Une preuve que les archives peuvent être un lieu patrimonial vivant et jouant un rôle essentiel dans la vie de la cité. Lieu de conservation de la mémoire collective, il a également vocation à interroger l'identité d'aujourd'hui à la lumière de celle d'hier.

La démarche de Saint-Étienne cosmopolitaine n'est pas sans rappeler celle du Centre mémoire et société, le Rize à Villeurbanne. C'est d'ailleurs une ancienne « rizienne » qui a rejoint l'équipe au poste de responsable de l'action culturelle. En effet, l'avenir et la vocation des archives se situent bien dans le champ de la citoyenneté. Au-delà de la mise à disposition de ressources, les archives peuvent devenir un carrefour d'échanges, un outil de questionnement et de médiation.

Aujourd'hui, dans le cadre des réflexions autour d'un nouveau bâtiment pour les archives (saturées depuis trois ans), la reconnaissance publique du rôle social et citoyen des archives constitue un élément central. L'équipement n'est plus envisagé aujourd'hui par les élus comme une simple réponse à des contraintes de stockage, mais comme pouvant répondre à des problématiques sociales et urbaines.

Saint-Étienne cosmopolitaine constituera sans doute une étape importante dans le développement des Archives municipales et aura marqué de manière significative le territoire. Ce projet fut précurseur sur de nombreux points : le travail sur l'héritage des migrations, la réinterrogation et la collecte de sources, la fédération de nombreux acteurs et une programmation inédite. Si la coordination du projet et des partenaires a constitué une lourde tâche, la reconnaissance fut réelle. Désormais, pour toute question sur l'histoire des migrations, les Archives municipales sont devenues le référent scientifique. Au-delà de la question du public, c'est le statut même des archives - comme objet et lieu - qui progressivement évolue vers plus de légitimité à porter des sujets de société. 